\title{
Development of single nanometer-sized ultrafine oxygen bubbles to overcome the hypoxia-induced resistance to radiation therapy via the suppression of hypoxia-inducible factor-1 $\alpha$
}

\author{
MISAKI IIJIMA $^{1 *}$, NAVCHAA GOMBODORJ $^{2 *}$, YOSHIAKI TACHIBANA ${ }^{3 *}$, KOHSUKE TACHIBANA $^{3 *}$, \\ TAKEHIKO YOKOBORI ${ }^{1}$, KYOKO HONMA $^{3}$, TAKASHI NAKANO ${ }^{2}$, TAKAYUKI ASAO $^{4}$, \\ RYUSUKE KUWAHARA ${ }^{5}$, KAZUHIRO AOYAMA ${ }^{5}$, HIDEHIRO YASUDA ${ }^{5}$, \\ MATTHEW KELLY $^{6}$, HIROYUKI KUWANO ${ }^{1}$ and DAI YAMANOUCHI ${ }^{6,7}$ \\ Departments of ${ }^{1}$ General Surgical Science, and ${ }^{2}$ Molecular Pharmacology and Oncology, Gunma University \\ Graduate School of Medicine, Maebashi, Gunma 371-8511; ${ }^{3}$ Sigma Technology Inc., Hitachinaka, Ibaraki 312-0053; \\ ${ }^{4}$ Big Data Center for Integrative Analysis, Gunma University Initiative for Advance Research (GIAR), Maebashi, \\ Gunma 371-8511; ${ }^{5}$ Research Center for Ultra-High Voltage Electron Microscopy, Osaka University, Ibaraki, \\ Osaka 567-0047, Japan; ${ }^{6}$ Division of Vascular Surgery, Department of Surgery, \\ University of Wisconsin School of Medicine and Public Health, Madison, WI 53792; \\ ${ }^{7}$ Wisconsin Nanobubble Research and Development Institute, Verona, WI 53593, USA
}

Received June 28, 2017; Accepted January 12, 2018

DOI: $10.3892 /$ ijo.2018.4248

\begin{abstract}
Radiation therapy can result in severe side-effects, including the development of radiation resistance. The aim of this study was to validate the use of oxygen nanobubble water to overcome resistance to radiation in cancer cell lines via the suppression of the hypoxia-inducible factor $1-\alpha$ (HIF-1 $\alpha$ ) subunit. Oxygen nanobubble water was created using a newly developed method to produce nanobubbles in the single-nanometer range with the $\Sigma$ PM- 5 device. The size and concentration of the oxygen nanobubbles in the water was examined using a cryo-transmission electron microscope. The nanobubble size was ranged from 2 to $3 \mathrm{~nm}$, and the concentration of the nanobubbles was calculated at $2 \times 10^{18}$ particles $/ \mathrm{ml}$. Cell viability and HIF- $1 \alpha$ levels were evaluated in EBC-1 lung cancer and MDA-MB-231 breast
\end{abstract}

Correspondence to: Dr Dai Yamanouchi, Division of Vascular Surgery, Department of Surgery, University of Wisconsin School of Medicine and Public Health, 600 Highland Avenue, G5/319, Madison, WI 53792, USA

E-mail: yamano@surgery.wisc.edu

Dr Takehiko Yokobori, Department of General Surgical Science, Gunma University Graduate School of Medicine, 3-39-22 Showamachi, Maebashi, Gunma 371-8511, Japan

E-mail: bori45@gunma-u.ac.jp

${ }^{*}$ Contributed equally

Key words: ultrafine nanobubble, hypoxia, hypoxia-inducible factor- $1 \alpha$, lung cancer, radiation sensitizer, nanobubble $\mathrm{O}_{2}$ cancer cells treated with or without the nanobubble water and radiation under normoxic and hypoxic conditions in vitro. The cancer cells grown in oxygen nanobubble-containing media exhibited a clear suppression of hypoxia-induced HIF-1 $\alpha$ expression compared to the cells grown in media made with distilled water. Under hypoxic conditions, the EBC-1 and MDA-MB231 cells displayed resistance to radiation compared to the cells cultured under normoxic cells. The use of oxygen nanobubble medium significantly suppressed the hypoxia-induced resistance to radiation compared to the use of normal medium at 2, 6, 10 and $14 \mathrm{~Gy}$ doses. Importantly, the use of nanobubble media did not affect the viability and radiation sensitivity of the cancer cell lines, or the non-cancerous cell line, BEAS-2B, under normoxic conditions. This newly created single-nanometer range oxygen nanobubble water, without any additives, may thus prove to be a promising agent which may be used to overcome the hypoxia-induced resistance of cancer cells to radiation via the suppression of HIF-1 $\alpha$.

\section{Introduction}

Progress in peri-operative management and adjuvant therapy has led to the improved survival of patients with lung cancer (1-3). It has been reported that adjuvant radiation therapy is effective and is often performed to eliminate lung cancer cells $(4,5)$. However, radiation therapy often induces radiation resistance and severe side-effects, including radiation-induced lung disease (RILD), which may range from treatable acute pneumonitis to lethal fibrosis (6-8). Therefore, overcoming resistance to radiation without inducing additional severe side-effects is vital for the treatment of patients with refractory lung cancer. 
Rapidly growing tumors located at a distance from the supporting vasculature results in the characteristic tumor microenvironment of low oxygen and nutrients $(9,10)$. The hypoxia-inducible factor- $1 \alpha(\mathrm{HIF}-1 \alpha)$ subunit is an important regulator of cellular oxygen homeostasis and hypoxia adaptation (11-13). In general, it has been reported that cancer cells under hypoxic conditions acquire resistance to radiation therapy, and to most types of chemotherapy in various types of cancer, via the accumulation of HIF-1 $\alpha$ (14-19).

High expression levels of HIF-1 $\alpha$ have been reported to be associated not only with radiation resistance, but also with a poor prognosis of patients with lung cancer (20-23). Shibamoto et al reported that the administration of a HIF-1 inhibitor, in adjunction with radiation, significantly suppressed the proliferation of lung cancer cell lines in vivo (24). However, some HIF-1 inhibitors may induce deleterious side-effects in non-cancerous tissues. Therefore, a therapeutic HIF-1-targeting strategy without side-effects may be an ideal radiation sensitizer for refractory cancers which are resistant to radiation in clinical practice.

Strategies for the treatment of hypoxia to overcome resistance to radiation have included the development of several radiosensitizers, and methods for directly increasing blood oxygenation, such as pure oxygen or carbogen breathing, ozone therapy, hyperbaric oxygen therapy, hydrogen peroxide injections and the administration of suspensions of oxygen carrier liquids, including ultrafine oxygen nanobubble water (25). However, these experimental models have shown limited success owing to unwanted side-effects and insufficient efficacy. To improve the therapeutic significance of HIF-1 $\alpha$ targeting, we focused on the creation of oxygen nanobubbles in a single-nanometer range. As previously indicated, smaller bubbles are more stable and possibly more effective in penetrating target cells (26).

In this study, we sought to validate a newly developed method to create oxygen nanobubble water in the single nanometer range, and to examine its effect on HIF-1 $\alpha$ expression and hypoxia-induced resistance to radiation across multiple cancer cell lines.

\section{Materials and methods}

Formation of nanobubble by $\Sigma P M-5$. Oxygen nanobubble water was prepared by a nanobubble water preparation device ¿PM-5 (bellows pump type) (27). In brief, oxygen and pure water were mixed at $0.4 \mathrm{MPa}$ and pushed out from the nozzle. The oxygenated water collided at high velocity to create nanobubble.

Characterization of nanobubble using a cryo-transmission electron microscope. The nanobubble water was diluted 100 -fold for measurement. Pure water with or without diluted nanobubble was rapidly frozen using Vitrobot Mark IV (FEI Co., Ltd., Hillsboro, OR, USA). The samples were embedded in amorphous ice for observation. The sample thickness was $200 \mathrm{~nm}$. Nanobubbles embedded in amorphous ice at a sample temperature of about $-193^{\circ} \mathrm{C}$ were directly observed using a cryo-transmission electron microscope Titan Krios (FEI Co., Ltd.). The electron beam used for observation is approximately 20 electrons $/ \AA^{2}$ by the low-dose technique, and there is almost no increase in sample temperature during photography.

Cell lines. The EBC-1 humanlung cancercell line was purchased from the RIKEN Cell Resource Center of Biomedical Research (Tsukuba, Japan), and the MDA-MB-231 human breast cancer cell line and BEAS-2S non-cancerous human bronchial cell line were from the American Type Culture Collection (Manassas, VA, USA). Baseline culture medium was prepared using RPMI-1640 medium (Wako, Osaka, Japan), which was dissolved in water with or without the oxygen nanobubble. The cells were cultured in filtered $(0.22 \mu \mathrm{m})$ nanobubble or normal RPMI-1640 supplemented with $10 \%$ fetal bovine serum and $1 \%$ penicillin and streptomycin antibiotics, and incubated at $37^{\circ} \mathrm{C}$ and $5 \% \mathrm{CO}_{2}$. For hypoxia, the cells were incubated under hypoxic conditions $\left(1 \% \mathrm{O}_{2}\right)$ using the BIONIX-1 hypoxic culture kit (Sugiyamagen, Tokyo, Japan) for $24 \mathrm{~h}$.

Analysis of cell viability under normoxic and hypoxic conditions. Cell viability was analyzed using the Cell Counting kit-8 (CCK-8; Dojindo Laboratories, Kumamoto, Japan). The cells were seeded $4 \times 10^{3} / 100 \mu 1$ per well in 96 -well plates. After $24 \mathrm{~h}$, normal RPMI-1640 medium was changed with meda with or without oxygen nanobubbles, and the cells were incubated under normoxic $\left(21 \% \mathrm{O}_{2}\right.$, room air) or hypoxic conditions $\left(1 \% \mathrm{O}_{2}\right)$. Following $24 \mathrm{~h}$ of incubation, the cells were irradiated at 2, 6, 10, and $14 \mathrm{~Gy}$ doses using an X-ray machine (Faxitron RX-650; Faxitron X-Ray LLC, Lincolnshire, IL, USA) with $100 \mathrm{kV}$, Al $0.3 \mathrm{~mm}$ filter. Following a 72-h incubation post-radiation, $10 \mu \mathrm{l}$ of $\mathrm{CCK}-8$ solution were added to each well and the plates were incubated at $37^{\circ} \mathrm{C}$ for $2 \mathrm{~h}$. The absorbance was detected at $450 \mathrm{~nm}$ using a plate reader (Bio-Rad, Hercules, CA, USA).

Protein extraction and western blot analysis. Protein extraction was performed using lysis buffer [10\% glycerol, $10 \mathrm{mM}$ Tris-HCl (pH 7.5), 1 mM EDTA, 400 mM NaCl, 0.5\% NP40, $4 \mu \mathrm{g} / \mathrm{ml}$ aprotonin, PMSF, proteasome inhibitor MG-132 and $1 \mathrm{mM}$ DTT]. Total protein $(10 \mu \mathrm{g})$ was electrophoresed on a $10 \%$ polyacrylamide gel, and then electroblotted at $300 \mathrm{~mA}$ for $90 \mathrm{~min}$ on a nitrocellulose membrane (Invitrogen, Carlsbad, CA, USA). Western blot analysis was used to confirm the protein expression of HIF-1 $\alpha$ and HSC70: These proteins were detected using anti-HIF-1 $\alpha$ rabbit polyclonal antibody $(1: 1,000)$ (Cell Signaling Technology, Cat. no. 3716) and anti-HSC70 mouse monoclonal antibody $(1: 1,000)$ (Santa Cruz Biotechnology, Cat. no. sc-7298). HSC70 expression was used as a loading control. The signals were detected using the ECL Select Western Blotting Detection System (GE Healthcare Life Sciences, Pittsburgh, PA, USA) and Image Quant LAS 4000 software (GE Healthcare Life Sciences).

Radiation treatment under normoxic and hypoxic conditions. Following a 24-h pre-incubation, the EBC-1 and MDA-MB231 cells in 96-well plates were exposed to hypoxic $\left(1 \% \mathrm{O}_{2}\right)$ and normoxic conditions with normal or oxygen nanobubble medium for 6 and $24 \mathrm{~h}$, and then treated with radiation. The $\mathrm{O}_{2}$ concentration was continuously evaluated using $\mathrm{O}_{2}$ concentration measuring devices (Oxy- $\mathrm{M} \mathrm{O}_{2}$ monitor, Jikco) in the bags. Cell viability was evaluated by CCK- 8 assay after $72 \mathrm{~h}$ 
A
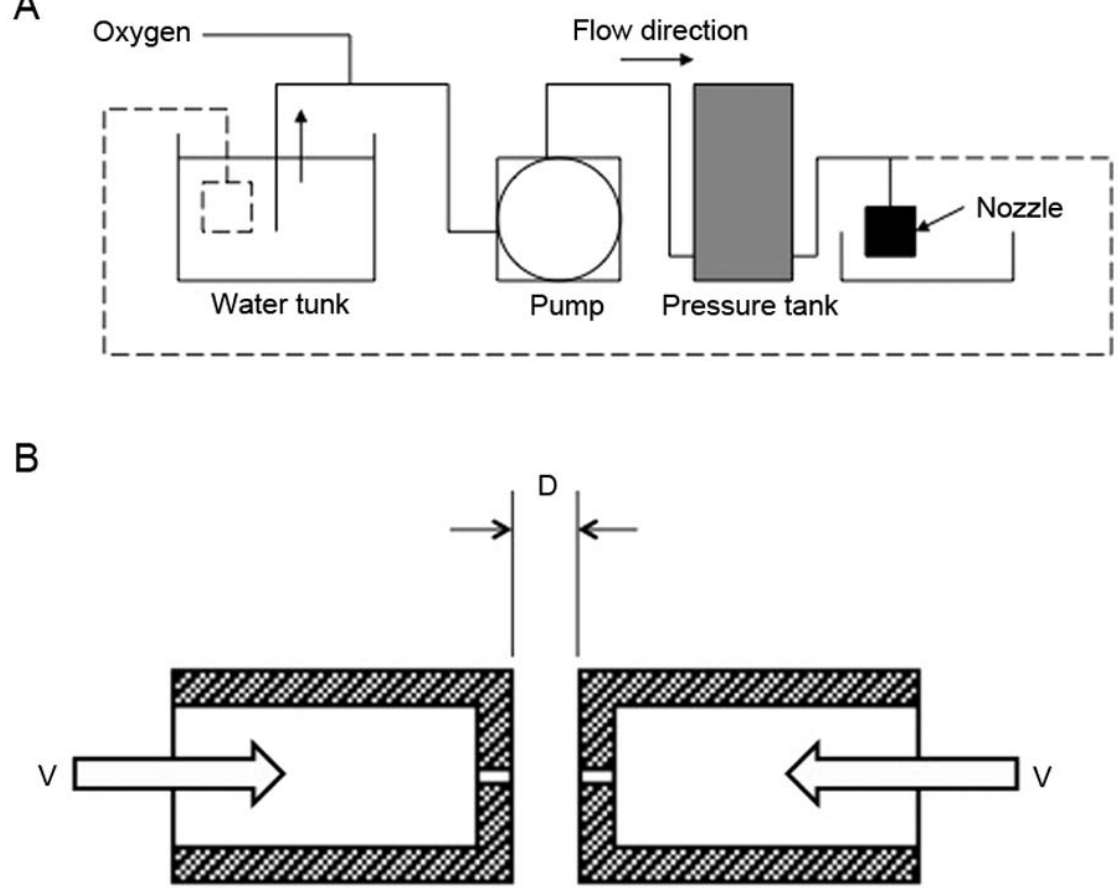

Figure 1. $\Sigma$ PM-5 nanobubble generator. (A) Schematic representation of $\Sigma$ PM-5. Water was pumped into the pressure tank where water and oxygen were mixed at 0.4 MPa. The oxygenated water was then pushed out through the small holes in the nozzle. (B) The schematic representation of the nozzle. Two small holes were placed horizontally in the nozzle. The pressurized and oxygenated water pushed out from these holes will collide to create the nanobubbles.

of incubation under normoxic conditions. An X-ray machine (Faxitron RX-650; Faxitron X-Ray LLC) with $100 \mathrm{kV}$, Al $0.3 \mathrm{~mm}$ filter was used as the radiation source for treatment.

Clonogenic assay under normoxic and hypoxic conditions. The cells plated into 6-well plates with RPMI-1640 normal medium and incubated at $37^{\circ} \mathrm{C}$ in humidified $5 \% \mathrm{CO}_{2}$ for $24 \mathrm{~h}$. Follwing a medium change, using the medium with or without oxygen nanobubble, the hypoxic plates were directly incubated under hypoxic conditions $\left(1 \% \mathrm{O}_{2}\right)$. After $24 \mathrm{~h}$, the cells were irradiated at various doses $(0,2,6,10$, and 14 Gy). After $72 \mathrm{~h}$, the medium was changed to normal RPMI-1640 medium and the cells were monitored every 3 days until colonies were visible. The plates were rinsed with phosphate-buffered saline (PBS), and the colonies were fixed with $99.5 \%$ ethanol and stained with $0.5 \%$ crystal violet (Sigma-Aldrich, St. Louis, MO, USA). The colonies counted up to at least 50 cells after staining. The surviva fraction (SF) was calculated as the mean (number of colonies counted/number of cells plated)/plating efficiency.

Statistical analysis. For continuous variables, the data are expressed as the means \pm standard deviation. Cell viability between the treatment groups was analyzed using JMP software (SAS Institute, Cary, NC, USA). A Student's t-test was used to compare the oxygen nanobubble group with the control group. A probability P-value $<0.05$ was considered to indicate a statistically significant difference.

\section{Results}

Characterization of $\Sigma P M-5$. The schematic representation of $\Sigma$ PM-5 is shown in Fig. 1A. The oxygen was mixed with the water in the pressurized tank at $0.4 \mathrm{MPa}$. The oxygenated water was then pumped out from the small hole (diameter, 0.3-0.6 $\mathrm{mm}$ ) in the nozzle (Fig. 1B). The velocity of water through the hole was calculated based on the flow rate measurement (Fig. 2A).

The high velocity water through the small hole will collide with the water from the other small hole placed horizontally. The energy of water collision was calculated as follows: $1 / 2 \mathrm{mV}^{2}+1 / 2 \mathrm{mV}^{2}=\mathrm{mV}^{2}$ (m, mass). The impact force was then calculated as follows: $\mathrm{F}=\mathrm{mV}^{2} / 1 / 2 \mathrm{D}(\mathrm{D}$, distance between the small hole). The collision energy force with the distance adjusted at $2 \mathrm{~mm}$ is shown in Fig. 2B.

Characterization of nanobubbles. The oxygen nanobubblecontaining water was created by $\Sigma$ PM-5 from pure water and oxygen. The nanobubble water was then diluted to 1:100 and embedded in amorphous ice. The samples were then observed using a cryo-transmission electron microscope. Pure water was used as the control. As shown in Fig. 3A, no particles appeared in amorphous ice prepared from pure water. As shown in Fig. 3B, the amorphous ice prepared from the oxygen nanobubble-containing water contained oxygen nanobubbles. In the area encircled by red broken lines, a dark contrast originating from the isolated nanobubbles was observed. The mean size was approximately 2-3 nm in diameter. On the other hand, in the area encircled by yellow broken lines, a necklace-like dark contrast originating from linear arrangement of nanobubbles was recognized. This result indicated that the nanobubbles partly aggregated. The volume of amorphous ice used for measurement was $1.8 \times 10^{-14} \mathrm{ml}(300 \times 300 \times 200 \mathrm{~nm}$ thickness) and contained around 360 bubbles inside. Since the nanobubble water sample was diluted 100 -fold, the particle number of oxygen nanobubbles was calculated to be $2 \times 10^{18}$ particles $/ \mathrm{ml}$. 
A

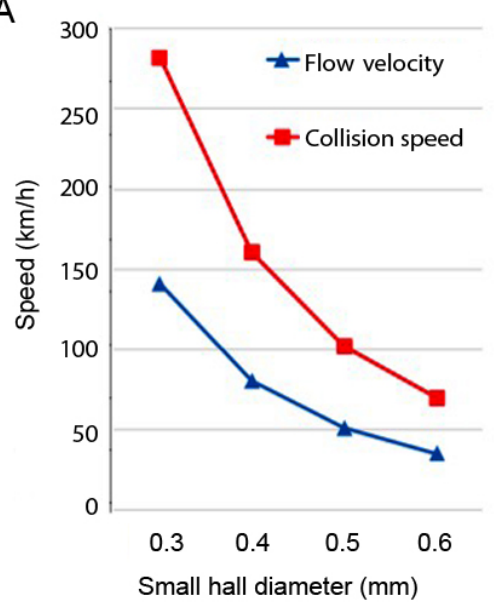

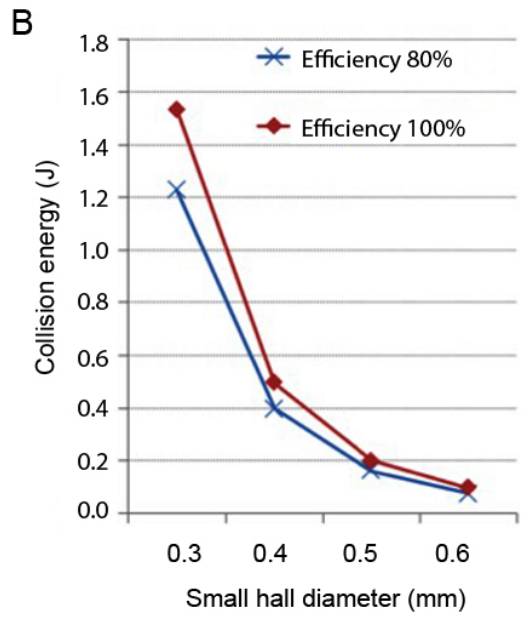

Figure 2. Speed and energy of water in the $\Sigma$ PM-5 nozzle. (A) The calculated speed of water collision based on the diameter of the small holes in the nozzle. Flow velocity through the small holes (blue) and the collision speed of water in the nozzle (red) are shown. (B) The calculated collision energies of water in the nozzle are shown.
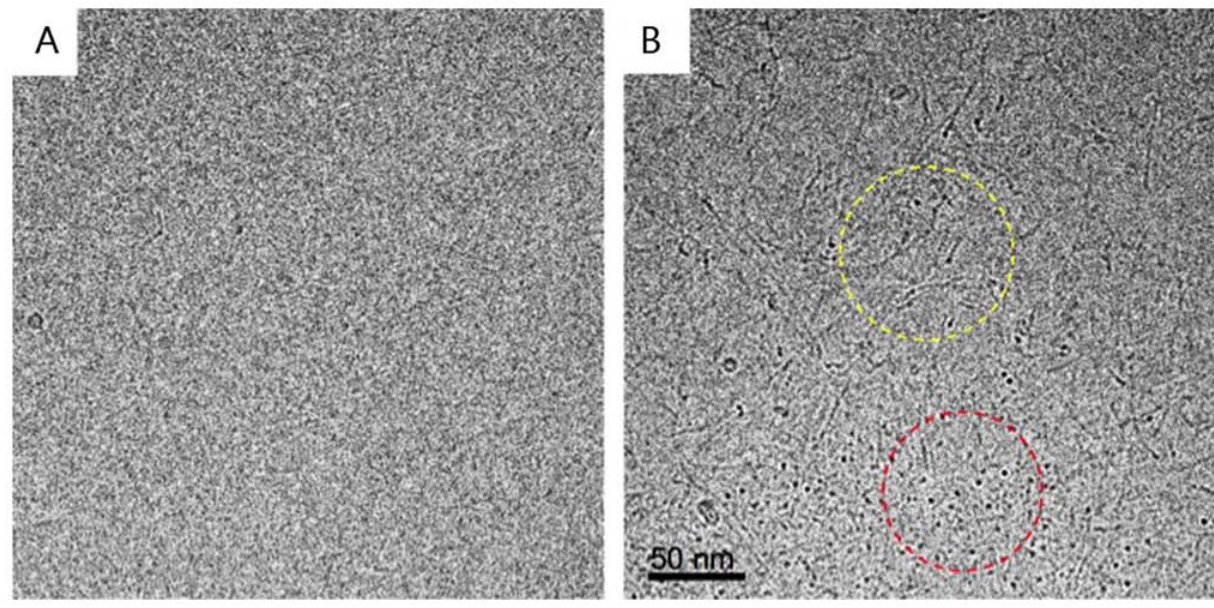

Figure 3. Characterization of nanobubbles produced by the $\Sigma$ PM-5 device. (A) Representative image of amorphous ice prepared from pure water by cryotransmission electron microscopy. No contrast from nanobubbles appears in the image. (B) Representative image of oxygen nanobubble water. Nanobubbles are visible as darker spots in the image. The area encircled in red highlights isolated nanobubbles, and the area encircled in yellow highlights linear arrangement of nanobubbles. Scale bar, $50 \mathrm{~nm}$.

Oxygen nanobubble medium suppresses hypoxia-induced HIF-1 $\alpha$ expression. The overexpression of HIF-1 $\alpha$ correlates with the resistance of cells to radiation $(21,22)$. We thus examined whether treatment with oxygen nanobubble medium can overcome the hypoxia-induced resistance of human cancer cells to radiation. The EBC-1 and MDA-MB-231 cells were pre-incubated in medium with or without oxygen nanobubbles for $24 \mathrm{~h}$, and were then analyzed for HIF-1 $\alpha$ expression after 6 and $24 \mathrm{~h}$ of exposure to hypoxic conditions. We found that the oxygen nanobubble water clearly suppressed hypoxia-induced HIF-1 $\alpha$ expression in the EBC-1 and MDA-MB-231 cells (Fig. 4A). We validated that this hypoxic condition induced the resistance of both the EBC-1 and MDA-MB231 cells to radiation (Fig. 4B).

Oxygen nanobubble medium alters cancer cell viability upon exposure to hypoxic conditions and radiation treatment. We treated the EBC-1 and MDA-MB-231 cells with radiation at doses of $0,2,6,10$, and 14 Gy to examine their sensitivities to radiation under both normoxic and hypoxic conditions (Fig. 5). Under hypoxic conditions, we demonstrated that our oxygen nanobubble medium enhanced the sensitivity of the EBC-1 cells to radiation compared to the normal medium at 2, 6, 10, and 14 Gy doses of radiation. as evidenced by a decreased cell viability upon oxygen nanobubble treatment (Fig. 5A, left panel). This effect was not significant in the MDA-MB-231 cells; however, we observed a similar tendency in these cells as in the EBC-1 cells (Fig. 5A, right panel). On the other hand, colony formation assay revealed that the oxygen nanobubble medium significantly suppressed the hypoxia-induced resistance of both the EBC-1 and MDA-MB231 cells to radiation compared to the normal medium at 2, 6, 10, and 14 Gy doses of radiation (Fig. 5B).

Normoxic application of oxygen nanobubble medium does not affect the survival or radiosensitivity of lung cancer, breast cancer, or non-cancer cell lines. The viability of the EBC-1 lung cancer, MDA-MB-231 breast cancer and non-cancerous 
A

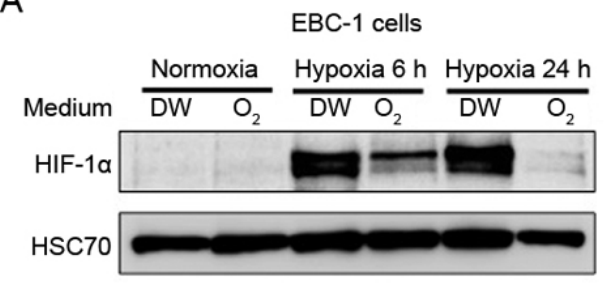

B

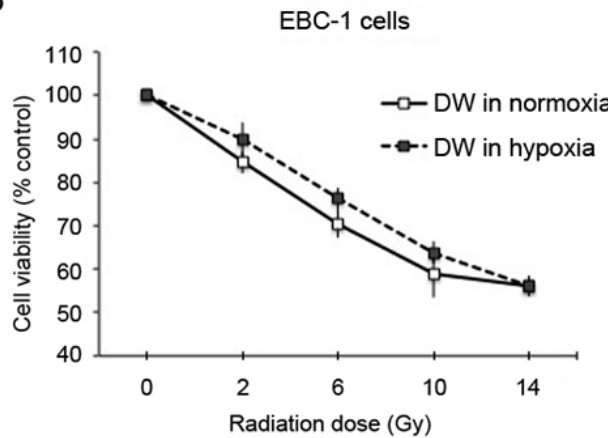

MDA-MB-231 cells

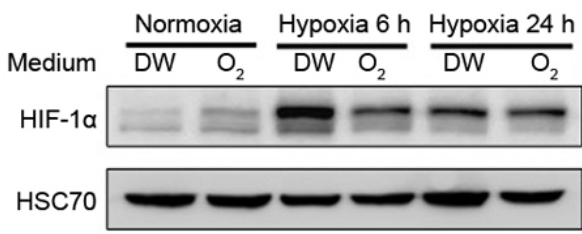

MDA-MB-231 cells

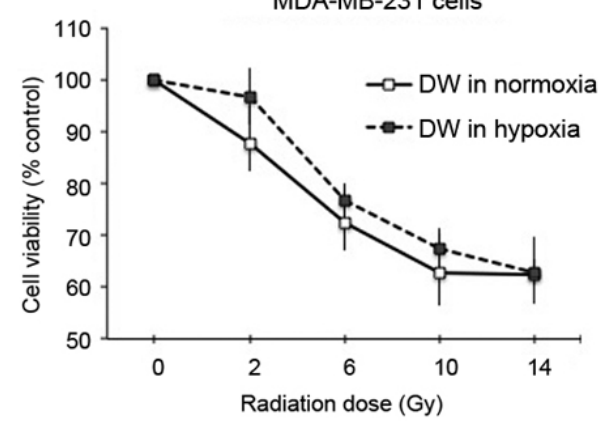

Figure 4. Oxygen nanobubble water suppresses HIF-1 $\alpha$ accumulation in hypoxic cancer cells. (A) HIF-1 $\alpha$ and HSC70 protein expression in the EBC-1 lung cancer cell line and MDA-MB-231 breast cancer cell line was evaluated by western blot analysis after 6 and 24 h of exposure ot hypoxia. Oxygen nanobubble medium clearly suppressed HIF-1 $\alpha$ induction under hypoxic conditions. (B) Hypoxia-induced radiation resistance was validated in both the EBC-1 and MDA-MB-231 cells. DW, normal medium; $\mathrm{O}_{2}$, oxygen nanobubble medium.

A

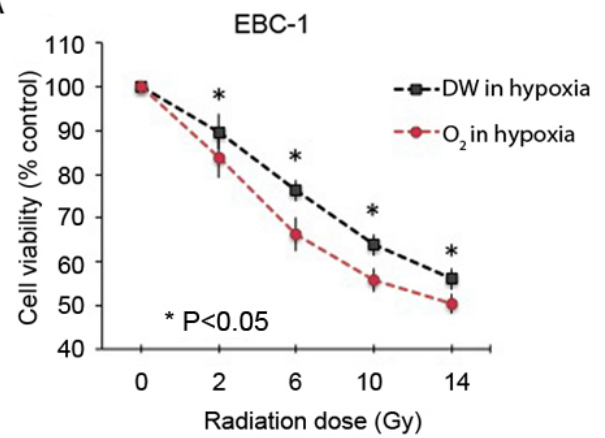

B

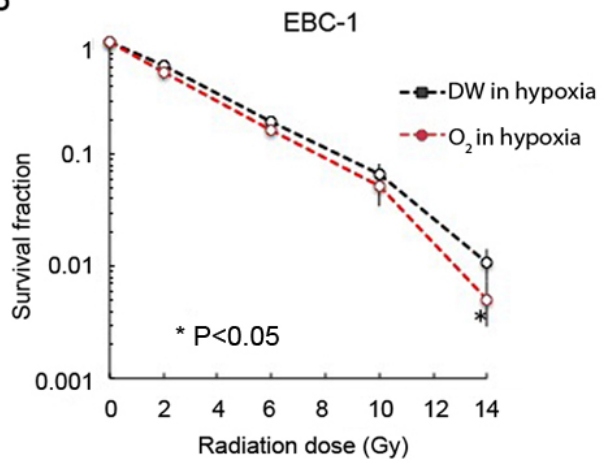

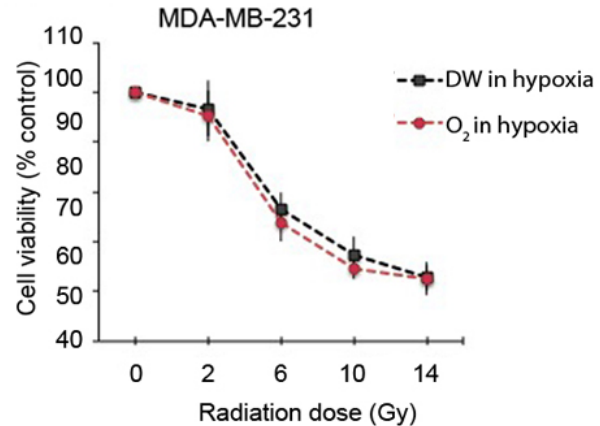

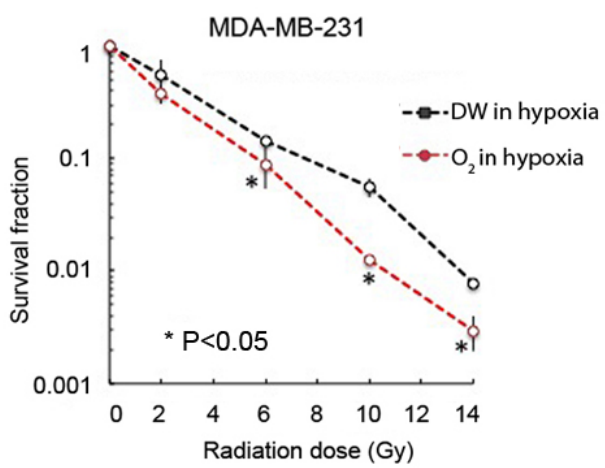

Figure 5. Oxygen nanobubble medium reverses hypoxia-induced radiation resistance in EBC-1 lung cancer and MDA-MB-231 breast cancer cells. (A) Cell viability assay showed that oxygen nanobubble medium suppressed hypoxia-induced radiation resistance in EBC-1 cells. A similar effect was observed with the MDA-MB-231 cells, although this was not significant; however, a similar tendency was validated. (B) Clonogenic assay revealed that oxygen nanobubble medium suppressed the hypoxia-induced resistance of EBC-1 and MDA-MB-231 cells to radiation. DW, normal medium; $\mathrm{O}_{2}$, oxygen nanobubble medium.

BEAS-2B bronchial cells was not affected by treatment with oxygen nanobubble medium under normoxic conditions (Fig. 6A). Furthermore, the sensitivity of the EBC-1,
MDA-MB231 and BEAS-2B cells to radiation was not affected by oxygen nanobubble treatment under normoxic conditions (Fig. 6B). 
A

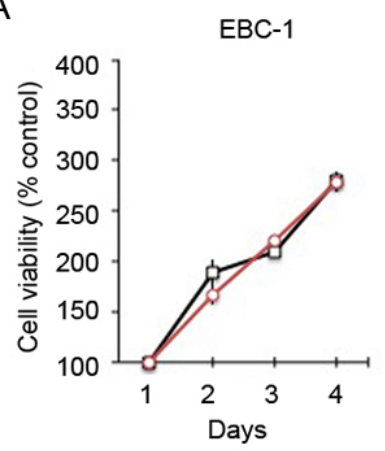

B

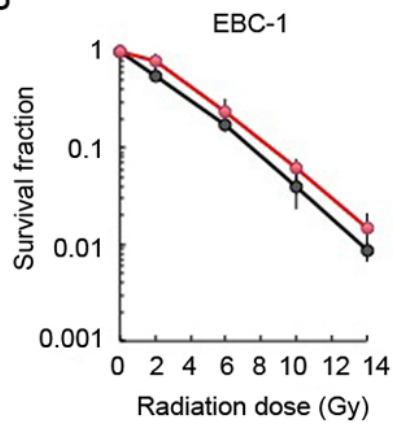

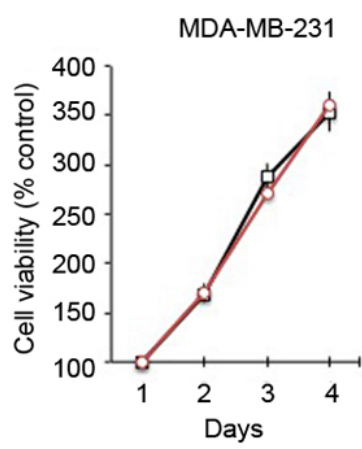

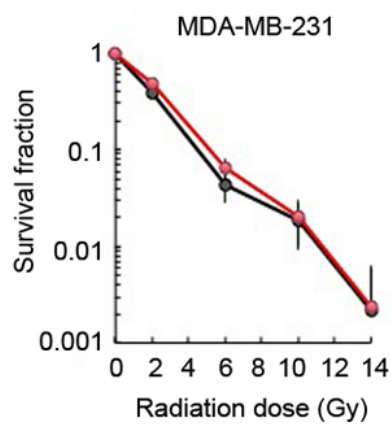

BEAS-2B
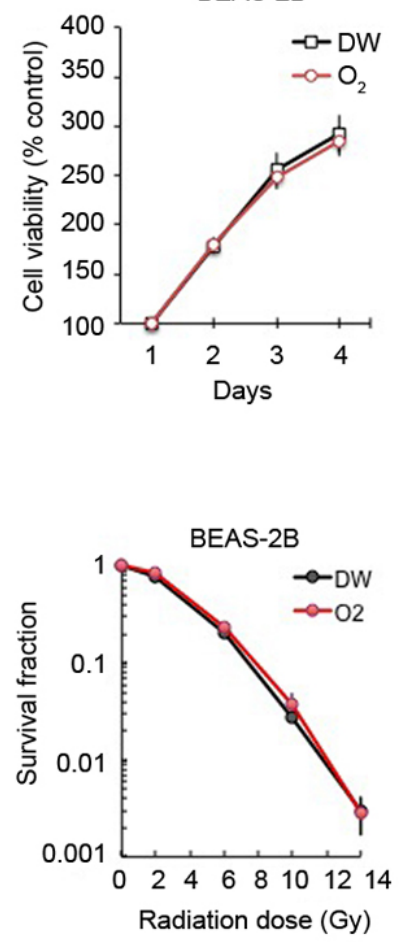

Figure 6. Oxygen nanobubble medium treatment under normoxic conditions is non-toxic to cancer and non-cancer cell lines. (A) CCK8 assay revealed that oxygen nanobubble medium did not reduce the viability of EBC-1 lung cancer, MDA-MB-231 breast cancer, or non-cancerous BEAS-2B bronchial cells compared to normal medium. (B) Clonogenic assay revealed that oxygen nanobubble medium did not affect the radiation sensitivity of EBC-1, MDA-MB-231, or BEAS-2B cells compared to normal medium.

\section{Discussion}

In this study, we produced oxygen nanobubble water at a single nanometer size using the nanobubble water preparation device, $\Sigma$ PM-5. The characterization of the oxygen nanobubbles using a cryo-transmission electron microscope verified that the nanobubbles were at the single-nanometer range. Moreover, the results of in vitro experiments demonstrated that the oxygen nanobubble water significantly enhanced the the sensitivity of human lung cancer and breast cancer cell lines to radiation under hypoxic conditions via the suppression of HIF-1 $\alpha$ expression.

Nanobubble water refers to a liquid containing small bubbles typically with $<200 \mathrm{~nm}$ in diameter (26). Unlike larger sized microbubbles which disappear relatively quickly, nanobubbles remain stable in water for a long period of time (28). Khier et al utilized oxygen gas filled particles to efficiently oxygenate human blood ex vivo without complement activation or hemolysis (29). Recently, Owen et al reported the delivery of oxygen through the oral administration of oxygen nanobubbles (25). Both research groups utilized lipid or surfactant to create and stabilize oxygen nanobubbles in a 50-200 $\mathrm{nm}$ range. In this study, we reported a newly developed method to create oxygen containing nanobubbles in the single-nanometer range. By utilizing a novel water hammer method, in which the pressurized oxygen saturated water collides in a high velocity, we produced oxygen-containing nanobubble water without any additives. The results from cryo-transmission electron microscopy measurement revealed stable oxygen nanobubbles in the single-nanometer range. To the best of our knowledge, this is the first study to demonstrate the creation and characterization of single nanometer-sized nanobubbles.

Resistance to radiation causes serious complications for patients with lung cancer. This resistance has been reported to be induced by several pathways, including those associated with hypoxia, tyrosine kinase receptors, AKT serine/threonine kinases, DNA damage repair, developmental pathways, adhesion pathways and inflammation $(30,31)$. The decreased oxygen concentration, and subsequent increase in HIF-1 $\alpha$ activity, is known to be associated with resistance to radiation, cell survival, angiogenesis and the proliferative activity of cancer cells (32-34). Therefore, in this study, we focused on the use of oxygen nanobubbles as a method to reoxygenate hypoxic cells and downregulate HIF-1 $\alpha$ activity. Several downstream targets of HIF, such as mediators of angiogenesis, including vascular endothelial growth factor (VEGF), cell survival regulators including insulin-like growth factor (IGF)-related factors, and cell proliferation regulators, such as c-MYC and insulin-like growth factor 2 (IGF2), are strongly associated with resistance to radiation and cancer aggressiveness under hypoxic conditions (35). In this study, we demonstrated the ability of our oxygen nanobubble preparation to significantly reduce HIF-1 $\alpha$ activity under hypoxic conditions; therefore, it may also modulate several of the important downstream mediators of radiation resistance and malignancy previously described.

This study utilized our oxygen nanobubble water as a modulator of radiation sensitivity under hypoxic conditions. This nanobubble water included only water and single nanometer-range oxygen bubbles, with an average size of 2-3 nm, without any chemical compounds. Small size bubbles have 
some advantages, including high stability and high oxygen occupancy compared to larger ones. On the other hand, the continuous administration of a high oxygen concentration is known to induce oxygen toxicity due to the production of reactive oxygen species $(36,37)$. However, our nanobubble water did not affect the viability of human lung cancer, breast cancer, or non-cancerous bronchial cells under normoxic conditions. Additionally, the $\mathrm{O}_{2}$ concentration-measuring devices demonstrated that the low oxygen concentration of our experimental hypoxia bags was not altered by the oxygen nanobubble medium during exposure to hypoxia. Therefore, our data suggests that our nanobubble water functions to modulate intracellular hypoxia in cancer cells via the suppression of hypoxia-induced HIF-1 $\alpha$ expression in spite of continuous hypoxic culture conditions.

Neo-adjuvant radiation and chemoradiotherapy (CRT) have been considered as effective therapeutic tools to accomplish radical resection and down-staging in patients with lung cancer $(38,39)$. Complete response is estimated as $14-40 \%$ by CRT $(40,41)$; on the other hand, patients with lung cancer that develop disease refractory to these therapies often have poor outcomes (42). Radiation is known to cause severe sideeffects, including lethal radiation fibrosis. However, modern radiation methodologies (including proton beam, heavy ion, stereotactic ablative body and intensity-modulated radiotherapies) are able to deliver precisely focused radiation to protect surrounding non-cancerous tissues, and effectively target refractory-prone hypoxic areas of tumors (43-45). Our data demonstrated that oxygen nanobubbles enhanced the sensitivity of human lung cancer cells and breast cancer cells to radiation under hypoxic conditions. In this study, we validated the efficacy of our oxygen nanobubble, across multiple tumor types, against radiation resistance and HIF-1 $\alpha$ accumulation under hypoxic conditions. Therefore, oxygen nanobubble water may serve as a sensitizing adjuvant when administered in combination with low dose radiation, which may enhance the efficacy of treatment, without increasing toxicity, in cancer patients.

In conclusion, in this study, we developed and characterized pure oxygen nanobubble water, in the single nanometer range, without any additives other than water and oxygen. In our human cancer cell-based experiments, oxygen nanobubble water demonstrated the ability to protect against hypoxia-induced radiation resistance through the suppression of HIF-1 $\alpha$. Our additive-free single nanometer-range oxygen nanobubble water may prove to be a promising modulator against hypoxia/HIF- $1 \alpha$-mediated radiation refractory cancers, although further studies are required in order to test its safety and effectiveness. Future studies are warranted to examine the preventative and therapeutic potential of our nanobubble water in mouse tumor models of radiation resistance. Additionally, an important challenge for future experiments will be to validate the stability of nanobubbles in vivo. Recently, Bandhari et al demonstrated the ability to detect oxygen nanobubbles via hyperspectral dark-field microscopy in live cells in vitro and tumor tissue ex vivo, and via ultrasound imaging of in vivo tumors $(46,47)$. Thus, we aim to employ these previously validated techniques for the detection of oxygen nanobubbles in tissue to examine the stability our oxygen nanobubbles in vivo. In addition, our in vitro experiments were performed in the presence of serum, therefore, the possibility exists that serum-specific cellular responses were elicited. However, we consider that serum may not be a critical factor to evaluate the relationship of hypoxia-induced radiation resistance and radiation sensitizers due to previous studies that have examined this relationship using serum-containing media (48-50). Nevertheless, to rule out any potential artifactual responses in our experiments due to the presence of serum, additional experiments are warranted under serum-free conditions.

\section{Acknowledgements}

This study was supported in part by the Japan Society for Promotion of Science (JSPS) Grant-in Aid for Scientific Research (Grant nos. 15K10085 and 22591450). A part of this study was supported by Osaka University Microstructural Characterization Platform as a program of 'Nanotechnology Platform' of the Ministry of Education, Culture, Sports, Science and Technology (MEXT), Japan. The authors are grateful to Mr. M. Nagata and Mr. K. Yoshiura in P.D.C.A. Inc. Japan for opening up new markets.

\section{Competing interests}

The authors M.I., N.G., T.Y., T.A., T.N., T.A., R.K., K.A., H.Y., H.K. and M.K., declare that they have no competing interests. Y.T., K.T., and K.H. are co-inventors of a patent (Tachibana Y, Tachibana K, Harada K, Sasajima S, Tamahashi K, Honma K, Matsumoto Y: METHOD, A BUBBLE GENERATING NOZZLE, AND AN APPARATUS FOR GENERATING MICRO-NANOBUBBLES JP.5555892,JP/PCT/2013/066902, Filed August 29, 2013; issued July 23, 2014), which has been filed in relation to the formulation of nanobubble described in the study. Y.T., K.T. and D.Y. are co-inventors of a patent (Yamannouchi D, Tachibana Y, Tachibana K: AQUEOUS SOLUTION CAPABLE OF BEING ADMINISTERED TO LIVING BODY, AND METHOD FOR PRODUCING SAME. WO 2017195852. JP/PCT2017/017779, Filed November 05, 2017) (Yamanouchi D, Tachibana Y, Tchibana K: AQUEOUS SOLUTION CONTAINING OZONE NANOBUBBLES, METHOD FOR PRODUCING SAME AND UTILIZATION OF AQUEOUS SOLUTION CONTAINING OZONE NANOBUBBLES. WO 2017199827. JP/PCT/2017/017781. Filed November 05, 2017.), which has been filed in relation to the formulation and application of nanobubble described in the study.

\section{References}

1. Nakagawa K, Watanabe SI, Kunitoh $\mathrm{H}$ and Asamura H; The Lung Cancer Surgical Study Group of the Japan Clinical Oncology Group: The Lung Cancer Surgical Study Group of the Japan Clinical Oncology Group: Past activities, current status and future direction. Jpn J Clin Oncol 47: 194-199, 2017.

2. Arriagada R, Auperin A, Burdett S, Higgins JP, Johnson DH, Le Chevalier T, Le Pechoux C, Parmar MK, Pignon JP, Souhami RL, et al; NSCLC Meta-analyses Collaborative Group: Adjuvant chemotherapy, with or without postoperative radiotherapy, in operable non-small-cell lung cancer: Two meta-analyses of individual patient data. Lancet 375: 1267-1277, 2010.

3. Fiteni F, Westeel V and Bonnetain F: Surrogate endpoints for overall survival in lung cancer trials: A review. Expert Rev Anticancer Ther 17: 447-454, 2017. 
4. Shafiq J, Hanna TP, Vinod SK, Delaney GP and Barton MB: A Population-based Model of Local Control and Survival Benefit of Radiotherapy for Lung Cancer. Clin Oncol (R Coll Radiol) 28 : 627-638, 2016

5. Yang CF, Chan DY, Speicher PJ, Gulack BC, Wang X, Hartwig MG, Onaitis MW, Tong BC, D'Amico TA, Berry MF, et al: Role of adjuvant therapy in a population-based cohort of patients with early-stage small-cell lung cancer. J Clin Oncol 34 1057-1064, 2016

6. Giridhar P, Mallick S, Rath GK and Julka PK: Radiation induced lung injury: Prediction, assessment and management. Asian Pac J Cancer Prev 16: 2613-2617, 2015.

7. Bradley $\mathbf{J}$ and Movsas B: Radiation pneumonitis and esophagitis in thoracic irradiation. Cancer Treat Res 128: 43-64, 2006.

8. Marks LB, Yu X, Vujaskovic Z, Small W Jr, Folz R and Anscher MS: Radiation-induced lung injury. Semin Radiat Oncol 13: 333-345, 2003.

9. Bertout JA, Patel SA and Simon MC: The impact of $\mathrm{O}_{2}$ availability on human cancer. Nat Rev Cancer 8: 967-975, 2008.

10. Kunz M and Ibrahim SM: Molecular responses to hypoxia in tumor cells. Mol Cancer 2: 23, 2003.

11. Ke Q and Costa M: Hypoxia-inducible factor-1 (HIF-1). Mol Pharmacol 70: 1469-1480, 2006.

12. Semenza GL: Hypoxia-inducible factors: Mediators of cancer progression and targets for cancer therapy. Trends Pharmacol Sci 33: 207-214, 2012

13. Keith B, Johnson RS and Simon MC: HIF1 $\alpha$ and HIF2 $\alpha$ : Sibling rivalry in hypoxic tumour growth and progression. Nat Rev Cancer 12: 9-22, 2011

14. Wilson WR and Hay MP: Targeting hypoxia in cancer therapy. Nat Rev Cancer 11: 393-410, 2011.

15. Harrison LB, Chadha M, Hill RJ, Hu K and Shasha D: Impact of tumor hypoxia and anemia on radiation therapy outcomes. Oncologist 7: 492-508, 2002

16. Brown JM and Wilson WR: Exploiting tumour hypoxia in cancer treatment. Nat Rev Cancer 4: 437-447, 2004

17. Shannon AM, Bouchier-Hayes DJ, Condron CM and Toomey D: Tumour hypoxia, chemotherapeutic resistance and hypoxiarelated therapies. Cancer Treat Rev 29: 297-307, 2003.

18. Shimoda LA and Semenza GL: HIF and the lung: Role of hypoxia-inducible factors in pulmonary development and disease. Am J Respir Crit Care Med 183: 152-156, 2011.

19. Goudar RK and Vlahovic G: Hypoxia, angiogenesis, and lung cancer. Curr Oncol Rep 10: 277-282, 2008

20. Chen X, Wang P, Guo F, Wang X, Wang J, Xu J, Yuan D, Zhang J and Shao C: Autophagy enhanced the radioresistance of non-small cell lung cancer by regulating ROS level under hypoxia condition. Int J Radiat Biol 93: 764-770, 2017.

21. Ren W, Mi D, Yang K, Cao N, Tian J, Li Z and Ma B: The expression of hypoxia-inducible factor-1 $\alpha$ and its clinical significance in lung cancer: A systematic review and meta-analysis. Swiss Med Wkly 143: w13855, 2013

22. Yang SL, Ren QG, Wen L and Hu JL: Clinicopathological and prognostic significance of hypoxia-inducible factor-1 alpha in lung cancer: A systematic review with meta-analysis. J Huazhong Univ Sci Technolog Med Sci 36: 321-327, 2016.

23. Hung JJ, Yang MH, Hsu HS, Hsu WH, Liu JS and Wu KJ Prognostic significance of hypoxia-inducible factor-1alpha, TWIST1 and Snail expression in resectable non-small cell lung cancer. Thorax 64: 1082-1089, 2009

24. Shibamoto Y, Sugie C, Ito M and Ogino H: The Japanese experiences with hypoxia-targeting pharmacoradiotherapy: From hypoxic cell sensitisers to radiation-activated prodrugs. Expert Opin Pharmacother 5: 2459-2467, 2004

25. Owen J, McEwan C, Nesbitt H, Bovornchutichai P, Averre R, Borden M, McHale AP, Callan JF and Stride E: Reducing tumour hypoxia via oral administration of oxygen nanobubbles. PLoS One 11: e0168088, 2016.

26. Agarwal A, Ng WJ and Liu Y: Principle and applications of microbubble and nanobubble technology for water treatment Chemosphere 84: 1175-1180, 2011.

27. Tachibana Y, Tachibana K, Harada K, Sasajima S, Tamahashi K, Honma K and Matsumoto Y: Method, a bubble generating nozzle, and an apparatus for generating micro-nano bubbles. Sigma Technology, Inc, JP, PCT/JP2013/066902. Filed August 29, 2013 issued July 23, 2014.

28. Takahashi M, Chiba K and Li P: Free-radical generation from collapsing microbubbles in the absence of a dynamic stimulus. J Phys Chem B 111: 1343-1347, 2007.
29. Kheir JN, Polizzotti BD, Thomson LM, O'Connell DW, Black KJ, Lee RW, Wilking JN, Graham AC, Bell DC and McGowan FX: Bulk manufacture of concentrated oxygen gas-filled microparticles for intravenous oxygen delivery. Adv Healthc Mater 2: 1131-1141, 2013.

30. Vaupel P, Thews $\mathrm{O}$ and Hoeckel M: Treatment resistance of solid tumors: Role of hypoxia and anemia. Med Oncol 18: 243-259, 2001.

31. Kim BM, Hong Y, Lee S, Liu P, Lim JH, Lee YH, Lee TH, Chang KT and Hong Y: Therapeutic implications for overcoming radiation resistance in cancer therapy. Int J Mol Sci 16: 26880-26913, 2015.

32. Semenza GL: Targeting HIF-1 for cancer therapy. Nat Rev Cancer 3: 721-732, 2003.

33. Chan DA, Krieg AJ, Turcotte S and Giaccia AJ: HIF gene expression in cancer therapy. Methods Enzymol 435: 323-345, 2007.

34. Xia Y, Choi HK and Lee K: Recent advances in hypoxia-inducible factor (HIF)-1 inhibitors. Eur J Med Chem 49: 24-40, 2012.

35. Semenza GL: Hypoxia-inducible factor 1 and cancer pathogenesis. IUBMB Life 60: 591-597, 2008

36. Poprac P, Jomova K, Simunkova M, Kollar V, Rhodes CJ and Valko M: Targeting free radicals in oxidative stress-related human diseases. Trends Pharmacol Sci 38: 592-607, 2017.

37. Gào X and Schöttker B: Reduction-oxidation pathways involved in cancer development: A systematic review of literature reviews. Oncotarget 8: 51888-51906, 2017

38. Daly BD, Cerfolio RJ and Krasna MJ: Role of surgery following induction therapy for stage III non-small cell lung cancer. Surg Oncol Clin N Am 20: 721-732, 2011.

39. Xu YP, Li B, Xu XL and Mao WM: Is there a survival benefit in patients with stage IIIA (N2) non-small cell lung cancer receiving neoadjuvant chemotherapy and/or radiotherapy prior to surgical resection: A systematic review and meta-analysis. Medicine (Baltimore) 94: e879, 2015.

40. Baker S, Dahele M, Lagerwaard FJ and Senan S: A critical review of recent developments in radiotherapy for non-small cell lung cancer. Radiat Oncol 11: 115, 2016.

41. Campbell BA, Ball D and Mornex F: Multidisciplinary lung cancer meetings: Improving the practice of radiation oncology and facing future challenges. Respirology 20: 192-198, 2015.

42. Scagliotti GV, Novello S, Rapetti S and Papotti M: Current stateof-the-art therapy for advanced squamous cell lung cancer. Am Soc Clin Oncol Educ Book 33: 354-358, 2013.

43. Haasbeek CJ, Slotman BJ and Senan S: Radiotherapy for lung cancer: Clinical impact of recent technical advances. Lung Cancer 64: 1-8, 2009.

44. Cascales A, Martinetti F, Belemsagha D and Le Pechoux C: Challenges in the treatment of early non-small cell lung cancer: What is the standard, what are the challenges and what is the future for radiotherapy? Transl Lung Cancer Res 3: 195-204, 2014.

45. Huo M, Gorayski P, Pinkham MB and Lehman M: Advances in radiotherapy technology for non-small cell lung cancer: What every general practitioner should know. Aust Fam Physician 45: 805-809, 2016.

46. Bhandari P, Wang X and Irudayaraj J: Oxygen nanobubble tracking by light scattering in single cells and tissues. ACS Nano 11: 2682-2688, 2017

47. Bhandari PN, Cui Y, Elzey BD, Goergen CJ, Long CM and Irudayaraj J: Oxygen nanobubbles revert hypoxia by methylation programming. Sci Rep 7: 9268, 2017.

48. Luo H, Wang L, Schulte BA, Yang A, Tang S and Wang GY: Resveratrol enhances ionizing radiation-induced premature senescence in lung cancer cells. Int J Oncol 43: 1999-2006, 2013.

49. Millet P, Granotier C, Etienne O and Boussin FD: Radiationinduced upregulation of telomerase activity escapes PI3-kinase inhibition in two malignant glioma cell lines. Int $\mathrm{J}$ Oncol 43: 375-382, 2013.

50. Lechtman E and Pignol JP: Interplay between the gold nanoparticle sub-cellular localization, size, and the photon energy for radiosensitization. Sci Rep 7: 13268, 2017.

This work is licensed under a Creative Commons Attribution-NonCommercial-NoDerivatives 4.0 International (CC BY-NC-ND 4.0) License. 